Western University

Scholarship@Western

Brain and Mind Institute Researchers'

Publications

Brain and Mind Institute

$11-1-2017$

\title{
Spatial structure normalises working memory performance in Parkinson's disease
}

Sean J. Fallon

MRC Cognition and Brain Sciences Unit

Daniel Bor

University of Sussex

Adam Hampshire

MRC Cognition and Brain Sciences Unit

Roger A. Barker

John van Geest Centre for Brain Repair

Adrian M. Owen

MRC Cognition and Brain Sciences Unit, uwocerc@uwo.ca

Follow this and additional works at: https://ir.lib.uwo.ca/brainpub

Citation of this paper:

Fallon, Sean J.; Bor, Daniel; Hampshire, Adam; Barker, Roger A.; and Owen, Adrian M., "Spatial structure normalises working memory performance in Parkinson's disease" (2017). Brain and Mind Institute Researchers' Publications. 968.

https://ir.lib.uwo.ca/brainpub/968 


\title{
Research report
}

\section{Spatial structure normalises working memory performance in Parkinson's disease}

\author{
Sean J. Fallon ${ }^{a, b, *}$, Daniel Bor ${ }^{c, d, e}$, Adam Hampshire ${ }^{a, f}$, \\ Roger A. Barker ${ }^{g}$ and Adrian M. Owen ${ }^{a, h}$ \\ ${ }^{a}$ Medical Research Council Cognition and Brain Sciences Unit, Cambridge, UK \\ ${ }^{b}$ Department of Experimental Psychology, University of Oxford, UK \\ c Department of Informatics, University of Sussex, UK \\ d Sackler Centre for Consciousness Science, University of Sussex, UK \\ e Department of Psychology, University of Cambridge, UK \\ ${ }^{\mathrm{f}}$ Department of Medicine \& Centre for Neurotechnology, Imperial College London, UK \\ g Centre for Brain Repair, Department of Clinical Neurosciences, University of Cambridge, UK \\ $\mathrm{h}$ The Brain and Mind Institute, University of Western Ontario, Canada
}

\section{A R T I C L E I N F O}

Article history:

Received 17 March 2017

Reviewed 31 May 2017

Revised 28 July 2017

Accepted 19 August 2017

Action editor Sonja Kotz

Published online 1 September 2017

Keywords:

Parkinson's disease

Working memory

Chunking

Irrelevance

Attention

\begin{abstract}
A B S T R A C T
Cognitive deficits are a frequent symptom of Parkinson's disease (PD), particularly in the domain of spatial working memory (WM). Despite numerous demonstrations of aberrant WM in patients, there is a lack of understanding about how, if at all, their WM is fundamentally altered. Most notably, it is unclear whether span - the yardstick upon which most WM models are built - is compromised by the disease. Moreover, it is also unknown whether WM deficits occur in all patients or only exist in a sub-group who are executively impaired. We assessed the factors that influenced spatial span in medicated patients by varying the complexity of to-be-remembered items. Principally, we manipulated the ease with which items could enter - or be blocked from - WM by varying the level of structure in memoranda. Despite having similar levels of executive performance to controls, PD patients were only impaired when remembering information that lacked spatial, easy-tochunk, structure. Patients' executive function, however, did not influence this effect. The ease with which patients could control WM was further examined by presenting irrelevant information during encoding, varying the level of structure in irrelevant information and manipulating the amount of switching between relevant and irrelevant information. Disease did not significantly alter the effect of these manipulations. Rather, patients' executive performance constrained the detrimental effect of irrelevant information on WM. Thus, PD patients' spatial span is predominantly determined by level of structure in to-beremembered information, whereas their level of executive function may mitigate against the detrimental effect of irrelevant information.
\end{abstract}

(C) 2017 Elsevier Ltd. All rights reserved.

\footnotetext{
* Corresponding author. Department of Experimental Psychology, University of Oxford, OX13UD, UK.

E-mail address: sean.fallon@psy.ox.ac.uk (S.J. Fallon).
} 


\section{Introduction}

Cognitive deficits, in addition to the characteristic motoric problems, are a frequent symptom of Parkinson's disease (PD; Brown \& Marsden, 1988; Cools, 2006; Kehagia, Barker, \& Robbins, 2012; Monchi, Hanganu, \& Bellec, 2016; Owen, 2004). Aberrant performance on working memory (WM) tasks form a core component of these deficits (Bublak, Müller, Grön, Reuter, \& von Cramon, 2002; Cools, Miyakawa, Sheridan, \& D'Esposito, 2010; Lewis, Slabosz, Robbins, Barker, \& Owen, 2005; Poewe, Berger, Benke, \& Schelosky, 1991), with impairments most robustly seen in the spatial domain (Owen, Iddon, Hodges, Summers, \& Robbins, 1997; Postle, Jonides, Smith, Corkin, \& Growdon, 1997). Deficits on these tasks are largely thought to occur due to the dopaminergic abnormalities that characterise the disease (Sawamoto et al., 2008).

However, despite these demonstrations, we still lack an understanding of whether the basic architecture of WM - how information is encoded, stored and recalled - is altered in PD. The amount of information that can be recalled - WM span is the basic currency in which different mnemonic models are constructed, compared and evaluated (Fallon, Zokaei, \& Husain, 2016). Measures of WM span are also related to measures of real-world success (Gathercole, Brown, \& Pickering, 2003). Thus, any exploration of the architecture of WM deficits in PD should seek to identify the factors that determine WM span.

The Corsi block-tapping task is a common test of spatial span (Milner, 1971). The basic structure of this task is to require individuals to observe and encode a sequence of spatial locations and then, after a short delay, reproduce this sequence by touching the remembered locations. Although there have been several studies of spatial span in PD patients using Corsi-like tasks, the results have been mixed. Despite numerous demonstrations of intact spatial spans in early medicated $\mathrm{PD}$, reduced spans have also been reported (Fournet, Moreaud, Roulin, Naegele, \& Pellat, 2000; Kemps, Szmalec, Vandierendonck, \& Crevits, 2005; Stoffers, Berendse, Deijen, \& Wolters, 2003). There are likely to be two principal reasons for this: a failure to control for the complexity of memoranda and cognitive heterogeneity (executive performance) in patients.

Here, we consider four factors that may influence the complexity of memoranda: level of structure in the to-beremembered information, presence of irrelevant information, structure in the irrelevant information and degree of switching that is required between relevant and irrelevant information. Firstly, with regards to structure, most studies that have examined spatial span in PD patients have failed to control for the extent to which the to-be-remembered sequences can be re-organised into familiar or regular structures, i.e., the extent to which information can be chunked (Miller, 1956). This factor has been found to be a key determinant of performance on span tasks, with higher spans and concomitant increases in dorsolateral prefrontal cortex (DLPFC) activity observed during the encoding of structured verses unstructured material (Bor \& Owen, 2007; Bor, Cumming, Scott, \& Owen, 2004; Bor, Duncan, Lee, Parr, \& Owen, 2006; Bor, Duncan, Wiseman, \& Owen, 2003). The failure to control for this factor may also lead to a misrepresentation of patients' mnemonic abilities, either because encoding easily-chunked information 'normalises' their span (due to it being easier), or, because they are unable to derive the normative enhancement in spatial span when encoding easily-chunked information. For example, patients with moderate Alzheimer's disease fail to show improvements in span when remembering structured material (Huntley, Bor, Hampshire, Owen, \& Howard, 2011).

Secondly, impaired span in PD patients may only appear when irrelevant information has to be ignored. This line of reasoning stems from observations that the basal ganglia particularly its modulation by dopamine - are thought to be essential for filtering out irrelevant information (Baier et al., 2010; Gruber, Dayan, Gutkin, \& Solla, 2006; McNab \& Klingberg, 2008). In line with this, PD patients' WM deficits have been found to be exacerbated by irrelevant information (Lee et al., 2010).

Thirdly, however, there may be a modulatory role of salience in influencing the detrimental effect of irrelevant information. As the mirror-image of what occurs when relevant information is structured, were irrelevant information to contain structure its salience may increase and thus be harder to ignore. Such an effect could be anticipated on the basis that PD patients have already been shown to have impaired capacity to ignore salient information in the attentional domain (Cools, Rogers, Barker, \& Robbins, 2010). Therefore, we sought to determine whether a similar effect can be detected in the mnemonic domain by varying the level of spatial structure in the irrelevant as well as the relevant information.

Finally, the detrimental effect of irrelevant information on patients' mnemonic performance may be contingent upon its prior relevance, especially given the established literature showing that this group has impairments in switching attention and task sets (Cools, Barker, Sahakian, \& Robbins, 2001; Fales, Vanek, \& Knowlton, 2006; Hayes, Davidson, Keele, \& Rafal, 1998; Owen et al., 1993; Pollux, 2004). Indeed, in support of this claim, Moustafa, Sherman, and Frank (2008) found that mnemonic impairments in $\mathrm{PD}$ patients became more pronounced when they had to remember previously irrelevant information. Therefore, as a final manipulation of the complexity of memoranda, this study varied the extent to which participants had to update their demarcation between relevant and irrelevant information by including a condition in which they had to switch to attending to previously irrelevant information (where the relevant and irrelevant information were defined by colour; Fig. 1).

A perennial problem in characterising cognitive performance in PD is patient heterogeneity (Owen, 2004). For example, only a subgroup of PD patients, in the absence of dementia, exhibit deficits on so-called executive tasks such as planning, WM and attention (Kehagia et al., 2012; Tremblay, Achim, Macoir, \& Monetta, 2013; Williams-Gray et al., 2013), though estimates of prevalence vary (Aarsland et al., 2010). Thus, the appearance of WM deficits in patients, and the resulting conclusions, may greatly depend upon the baseline executive performance level of that sample. One way to circumvent this problem is to test a larger group of patients with varying levels of executive performance. Performance on the Tower of London (TOL) task has been used to stratify 


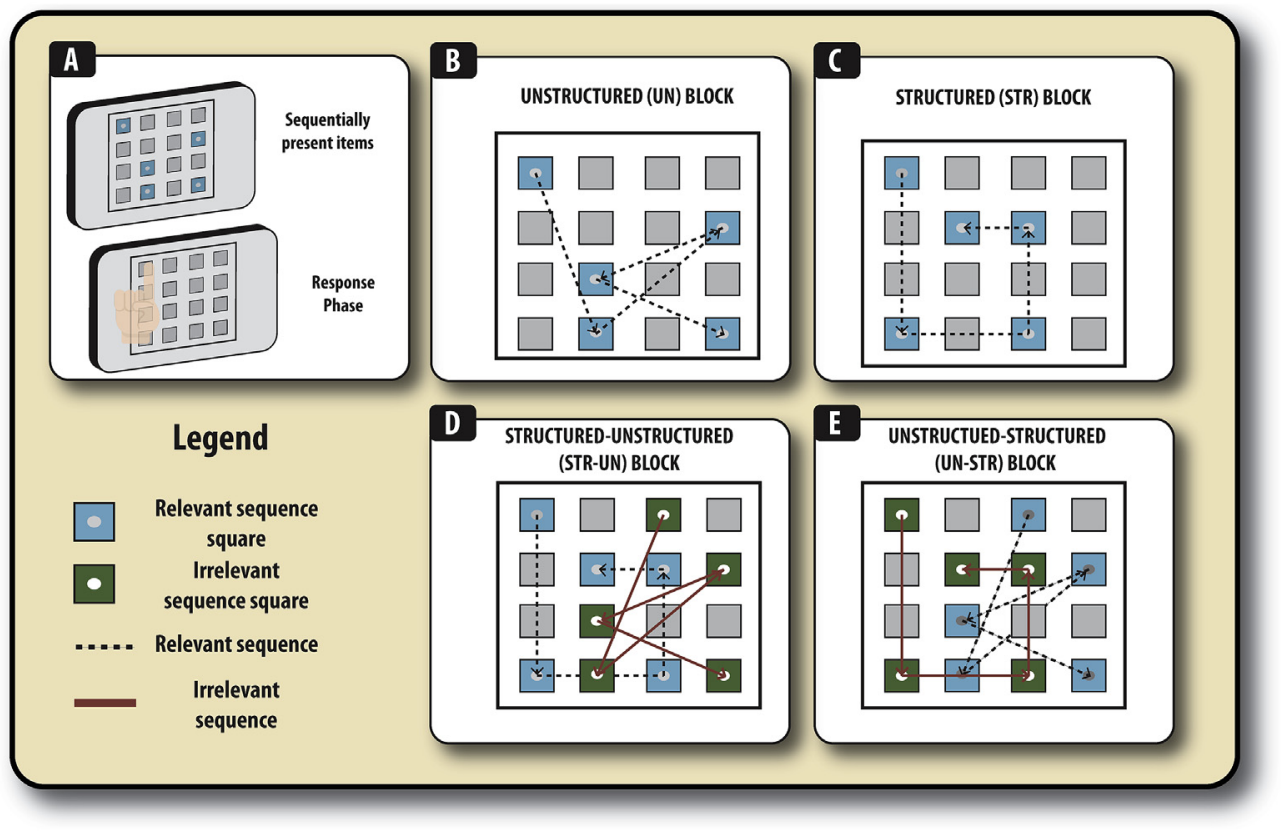

Fig. 1 - Illustration of the task and conditions. A) Schematic of the task protocol. Locations that had to be remembered were indicated by the grey squares on a $4 \times 4$ grid changing colour, sequentially. The number of locations was determined by the current set size. Then, after the presentation of auditory tone, participants had to reproduce the sequence in the order in which it appeared, by sequentially touching a square on the $4 \times 4$ grid. Example trials from four blocks (Fig. 1B-E). In the structured block (STR; Fig. 1C), the sequence of illuminated squares followed a predetermined rule which tended to produce sequences which were easy to group into smaller chunks. Unstructured (UN) sequences (Fig. 1B), however, were prevented from generating such patterns. In some blocks, participants were presented with two sequences: a relevant sequence that had to be remembered and an irrelevant sequence that had to be ignored. In the structured-unstructured (STR-UN) block (Fig. 1D), relevant information was structured and irrelevant information was unstructured. However, in the unstructured-structured (UN-STR; Fig. 1E) block, the relevant information was unstructured and the irrelevant information was structured. There was also an unstructured-unstructured block where neither type of information was structured (not shown).

patients into executively impaired and unimpaired groups (Fallon, Hampshire, Barker, \& Owen, 2016; Lewis, Cools et al., 2003; Lewis, Dove, Robbins, Barker, \& Owen, 2003). These studies have revealed that executively impaired patients do not have a generic deficit with WM but only perform poorly when information in WM has to be manipulated and show a preserved ability to effectively maintain information (Lewis, Cools et al., 2003). However, whether this conclusion holds true across the variations in the complexity of memoranda mentioned above has yet to be investigated. It could be hypothesised that patients' level of executive functioning is a key moderating variable in influencing the effect that the complexity of memoranda has on their spatial spans. This factor may also have played a role in generating some findings using WM span tasks in patients. Recently, in a relatively small group of patients, varying the level of structure in the tobe-remembered information did not significantly affect WM span (Gruszka, Bor, Barker, Necka, \& Owen, 2016). One possibility is that - in addition to being potentially underpowered patient heterogeneity may have masked the appearance of any selective deficit in remembering unstructured material. Therefore, in this study we also sought to examine the extent to which PD patients' spatial span performance is determined by the level of structure in the to-be-remembered information, while also examining the modulatory role of executive performance.

\section{Methods}

\subsection{Participants}

The study complied with the 1975 Helsinki declaration and was approved by the local ethics committee (National research ethics committee - Norfolk 08/H0306/26). All subjects gave informed consent prior to participating in the study. PD patients and healthy older adults without a history of previous neurological complaints (e.g., stroke or head jury) or reported psychiatric illness were invited to take part in the study. All patients had a Mini Mental Status Score (MMSE) $\geq 28$. To ensure a degree of medication homogeneity, only patients that were taking some form of dopaminergic medication were included in this study. Furthermore, given the aim of this study was to examine the role of executive functioning in influencing WM, included participants had to have completed the TOL planning task from the Cambridge 
Neuropsychological Test Automated Battery (CANTAB). This test assesses general executive functioning and performance has been found to be provide a good index of cognition in PD patients (Fallon, Hampshire et al., 2016; Lewis, Cools et al., 2003).

Data from 35 healthy older adults and 38 patients were included in this study. The mean age for controls was 63.49 $(\mathrm{SD}=5.7)$ and for patients was $66.34(\mathrm{SD}=7.2)$. These two groups did not statistically differ in age $[\mathrm{t}(71)=1.86, p=.066]$. Patients were taking a variety of dopaminergic medications (see Supplementary Table). Equivalent levodopa dose was calculated using a standard algorithm [(Levodopa dose $\times 1.2$ if COMT inhibitor) $(\times 1.2$ if $10 \mathrm{mg}$ of Selegiline or $\times 1.1$ if $5 \mathrm{mg}$ of Selegiline $)+[$ Pramipexole $\times 400]+[$ ropinirole $\times 40]+$ [Cabergoline $\times 160$ ].

\subsection{Design and procedure}

Both groups performed other cognitive tasks prior to starting the spatial span task; PD performed an attention set-shifting task (data to be reported elsewhere), whereas controls performed the TOL test from the CANTAB test battery. PD patients did not need to complete this test battery on the testing day as they had already completed this battery as part of a prior clinical assessment.

Participants viewed the spatial span task on a $15^{\prime \prime}$ CTX touch-screen monitor (resolution: $1024 \times 768$ ). Participants were inducted into the task using written instructions and randomly assigned a colour and order assignment. After this, participants completed two practice blocks, one run of the unstructured block and one run of the structured block. The order in which each block was performed was counterbalanced. The initial sequence length of these practice blocks was 1 square and there were 7 trials in each block. After the practice session, participants completed the three phases of the experiment. The spatial span task required participants to memorise, and reproduce, a sequence of spatial locations that were illuminated on a $4 \times 4$ grid of grey squares against a black background (see Fig. 1). A coloured bar was present at the bottom of the screen to indicate the target colour. For each trial, a sequence of squares flashed in the relevant and irrelevant (where appropriate, see below) colour. Each square remained illuminated for $1000 \mathrm{msec}$ and flashes were separated by a $500 \mathrm{msec}$ interval. At the end of the sequence a short tone prompted participants to make their response (using their right index finger). Participants were given a fixed time window within which to respond, determined by the length of the sequence of squares participants were presented with $(1000 \mathrm{msec}+400 \mathrm{msec}$ for each square). At the end of each trial, participants were informed (on the screen) whether they had got the answer correct. This experiment used a ratchet design: correct trials led to an increase in sequence length for the next trial by one square and incorrect trials led to a decrease in sequence length by one square. This was done so as to estimate participants' span - amount of information that could be remembered - and because a previous study that did not find differences between patients and controls in remembering structured information only used a fixed set size, i.e., four items (Gruszka et al., 2016).
In total, there were 7 blocks, with 10 trials per block. The starting sequence length of each block was determined by the average sequence length of the previous block (rounded down). For the first block of the testing session, the starting sequence length was set to three. In all the conditions, no square (spatial location) could appear more than once within a sequence. Spatial span was measured for each condition, by taking the average length of the last five trials for each block.

Spatial span was assessed across three separate phases. The order of these phases was not randomised to prevent carry over effects which could be expected to be larger in patients given their reported deficits in cognitive switching. The first phase examined spatial span during the encoding of unstructured (UN block) and structured (STR block) material without any irrelevant material. Different target colours were used for each block, and the order in which participants completed these two blocks was counter-balanced. As in Bor et al. (2003), a structured sequence was generated by ensuring that the next square in the sequence was always from the same row, column or diagonal line as the previous square in the sequence (i.e., it could move like the queen piece in chess). However, unstructured sequences involved no such moves (see Fig. 1).

In the second phase of the experiment, two sequences appeared simultaneously on the screen: a target sequence and non-target irrelevant sequence (presented in different colours). The same square could feature in both the target and irrelevant sequence, but not simultaneously. The experimental manipulation in this phase consisted of varying the level of structure in the relevant sequence or in the irrelevant sequence. There were three blocks within this phase: a block where neither the relevant sequence nor the irrelevant sequence was structured (UN-UN block); a structured/unstructured block, where the relevant sequence was structured but the irrelevant sequence was unstructured (STR-UN block); and finally an unstructured/structured block, where the irrelevant sequence was structured but the relevant sequence was unstructured (UN-STR block). These are displayed in Fig. 1. A separate colour combination was used for each block. The order in which each block was completed and the colour assignment for each block was also counterbalanced across participants (12 different colours were used across the experiment).

The third and final phase of the experiment introduced a switching component into the task. Whereas in the previous two phases of the task the target colour remained constant through the duration of the block, in this phase of the experiment the target colour could change at several points during the block. This phase consisted of two blocks, which varied the level of structure in the irrelevant sequence. There was an unstructured/unstructured block (SW-UN-UN) and an unstructured/structured block (SW-UN-STR; similar to the previous phase of the experiment, but with the addition of the switching element). Again, the colour combinations of these two blocks differed, as did the order in which participants completed these two tasks. The colour assignments were also counter-balanced across participants, with each block having a different colour for any given subject.

The results were analysed (in SPSS 19.0) using two separate mixed analysis of variances (ANOVAs). These analyses 
examined two separate questions: i) whether the effect of introducing structure varied according to whether relevant or irrelevant information was present (UN, STR, UN-UN and STR-UN blocks), ii) whether the effect of structured information in the irrelevant sequence on spatial span varied according to the requirement to switch between relevant and irrelevant sequences (UN-UN, UN-STR, SW-UN-UN, SW-UN-STR).

\section{Results}

\subsection{PD patients and controls had equivalent levels of overall executive functioning}

There was no significant difference between patients and controls in the total number of problems solved on the TOL [t(71) $=.093, p=.926]$. Thus, patients and controls showed equivalent levels of executive performance.

\subsection{Structure normalises PD patients' span}

To examine the effect that irrelevant information and its interaction with structure in the relevant sequence had on spans, performance on the UN, STR, UN-UN and STR-UN blocks were analysed with a mixed ANOVA with repeated measures on structure in the relevant sequence (structured, unstructured), irrelevant information (present, absent) and disease as a between-subject variable. Level of executive function (performance on the TOL) was entered as a meancentred covariate.

Spans were higher when sequences were spatially structured $[F(1,68)=12.45, p=.001]$ and patients had significantly lower spatial spans than healthy controls $[F(1,68)=6.56$, $p=.013]$. However, there was a significant interaction between spatial structure and disease $[F(1,68)=6.97, p=.010]$. Patients only had significantly lower spans when encoding unstructured sequences [t(68) $=3.69, p=.0004]$, but were not significantly impaired when recalling structured sequences [t(68) $=1.02, p=.314]$. Moreover, while patients had significantly higher spans when recalling structured compared to unstructured sequences [ $\mathrm{t}(37)=4.62, p=.00004]$, this effect was absent in the healthy older adults $[\mathrm{t}(34)=.70, p=.49]$. Thus, encoding and maintaining structured information normalised patients' spans.

Across patients and controls, the presence of an irrelevant sequence did not significantly affect spans $[F(1,70)=3.60$, $p=.062]$. There was no significant interaction with disease $[F(1,68)=1.40, p=.24]$, level of executive performance $(F<1)$ or spatial structure $[F(1,68)=1.22, p=.27]$. There was no threeway interaction between disease, spatial structure and presence of irrelevant information $[F(1,68)=2.36, p=.129]$.

With regards to the other factors, executive functioning significantly affected overall spatial spans $[F(1,68)=11.04$, $p=.001]$, with higher executive performance being associated with higher spans. However, despite this tight coupling between executive performance and overall span performance, this covariate did not interact with the effect of spatial structure $(F<1)$. Thus, executive performance did not modulate the beneficial effects of structure or modulate patients' performance on this task.

\subsection{The beneficial effects of structure on span is modulated by the level of dopaminergic medication}

Supplementary analyses examined the effect of Unified Parkinson's Disease Rating Scale (UPDRS) motor score and level of dopaminergic medication in modulating the relationship between the above experimental factors in influencing span in PD patients. There was no significant effect of UPDRS score on span and neither did it significantly interact with any experimental factor ( Fs $<1$ ). Equivalent L-dopa dose was found to have a significant effect on patients' spatial span $[F(1,36)=4.78, p=.035]$, with higher doses being associated with lower spans. Notably, equivalent L-dopa dose was found to significantly interact with structure and the presence of irrelevant information $[F(1,36)=5.04, p=.031]$. This three-way interaction was due to levodopa dose having a significant positive correlation with the mnemonic gain for remembering structured versus unstructured material in the presence of irrelevant information correlation ( $\triangle$ STRUN, UNUN) with levodopa dose $[r(38)=.360, p=.026$; Fig. $2 \mathrm{~B}]$, but there was no correlation between the difference in the spans for the single sequence structured and unstructured blocks $(\triangle \mathrm{STR}, \mathrm{UN})$ with levodopa dose $[r(38)=-150, p=.343]$. Thus, medication seems to exert its effects through bolstering the performance gain induced by the existence of structure in to-be-remembered information, specifically when irrelevant information is present.

In summary, whereas patients and controls did not react differently to the introduction of irrelevant information, they did react differently to the introduction of spatial structure in to-be-remembered spatial sequences. Patients appeared to extract a mnemonic benefit from the introduction of spatial structure and were able to attain normal levels of performance. Also, although level of executive performance was associated with differences in spatial span, performance on the TOL did not modulate the effect of the other experimental variations.

\subsection{PD patients' span is not modulated by switching and the presence of irrelevant information}

The effect of introducing a switching component into the task in the presence of structured irrelevant information was assessed by comparing performance on the UN-UN, UN-STR, SW-UN-UN and SW-UN-STR blocks with a mixed ANOVA with repeated measures on structure in the irrelevant sequence (unstructured, structured) and switching (nonswitching, switching) with disease as a between-subject variable and executive performance as a covariate (meancentred). One healthy control subject and one PD patient had missing data for one or more of the cells involved in this analysis and were therefore excluded from the analysis.

Across these four conditions, patients had significantly lower spans than healthy older adults $[F(1,68)=13.79$, $p=.0004]$. However, patients' impairment varied significantly across the experimental factors; there was a three-way interaction between disease, switching and the presence/ absence of structure in the irrelevant sequence $[F(1,68)=5.91$, $p=.018]$. Breaking this interaction down, there was no significant interaction between switching and level of structure 


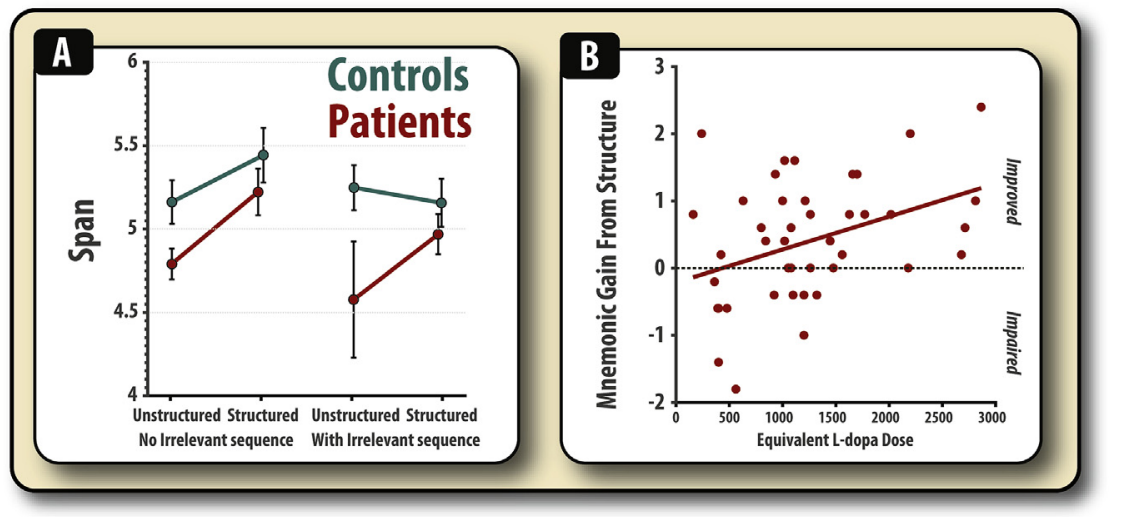

Fig. 2 - PD patients and controls spatial span. A) Span performance for PD patients and controls, split according to whether an irrelevant sequence was simultaneously presented and whether the relevant memoranda was structured or unstructured. PD patients were significantly impaired when remembering unstructured sequences but not structured sequences. Error bars reflect the standard error of the mean. B) The relationship between patients' equivalent levodopa medication dose (see method) and mnemonic gain from encoding structured sequences. The latter was calculated by subtracting spatial spans on unstructured blocks (UN and UN-UN) from structured blocks (STR and STR-UN). A higher score on this measure indicates patients' span improved when encoding structured compared to unstructured sequences.

in the irrelevant sequence for $\mathrm{PD}$ patients $(F<1)$. In contrast, in controls, these two factors significantly interacted $[F(1,33)=7.08, p=.012]$. This two-way interaction in controls was itself due to structure in the irrelevant sequence having opposite effects in switching and non-switching contexts. In non-switching contexts, irrelevant structure impaired spatial spans [t(33) $=2.17, p=.037$, whereas there was a trend towards controls having higher spans when irrelevant structure was present in switching contexts [ $\mathrm{t}(33)=1.88, p=.069]$. With regards to the main analysis, all other interactions with disease were not significant $(F s<1)$.

Similar to the previous section above, there was a significant effect of the level of executive functioning on spatial span scores $[F(1,68)=13.21, p=.001]$, with higher scores on the TOL being associated with higher spatial spans. In addition, there was a significant interaction between the level of executive performance and the effect of irrelevant information in influencing spatial spans $[F(1,68)=6.54, p=.013]$. This interaction was due to there being a significant positive correlation between executive performance and the mnemonic cost of structure in the irrelevant sequence $[r(71)=.296, p=.012]$, i.e., participants with higher levels of executive performance experienced a greater decrease in their spans by having to deal with structure in the irrelevant dimension. This pattern was only found to be significant in patients $[r(37)=.392, p=.016$; Fig. $3 B]$, but not controls $[r(34)=.078, p=.66]$. Level of executive performance did not significantly correlate with any other variable $(F s<1)$.

Thus, in summary, the above analysis yielded two main findings. Firstly, in controls but not patients, it appeared that the level of attention that was given to the relevant and irrelevant sequences (induced by switching) determined the beneficial or detrimental effect of irrelevant structure. When no switching occurred, irrelevant structure impaired spans, but when switching occurred, irrelevant structure improved spans. This effect was entirely absent in PD patients.
Secondly, in patients but not controls, the level of executive functioning appeared to influence the mnemonic cost of introducing structure into the irrelevant sequence; costs were most prominent in those patients with the higher level of executive functioning.

\subsection{Medication dose did not alter the effect of irrelevant information}

Supplementary analyses examined, separately, the effect of entering UPDRS motor score and equivalent L-dopa dose as covariates in the above analysis (for patients only). UPDRS did not have a significant main effect on span $[F(1,40)=1.44$, $p=.23$ ] and there was a trend towards UPDRS modulating the effect of switching on spatial span $[F(1,40)=3.67, p=.06]$ and no significant three-way interaction between UPDRS, switching and the structure in the irrelevant sequence $[F(1,40)=1.19$, $p=.28]$. For equivalent $\mathrm{L}$-dopa dose, there was a trend towards a main effect on $\operatorname{span}[F(1,41)=2.86, p=.098]$, but none of the other effects were significant $(F s<1)$.

\subsection{No evidence for differential fatigue in patients and controls}

One potential confound in our design is whether patients and controls experienced differential fatigue across the three phases study or merely responded different to the increasing difficult of the phases (addition of irrelevant information and switching).

To this end, we examined performance across the three phases of the experiment; phase 1 (single unstructured and structured sequences), phase 2 (unstructured with irrelevant unstructured sequences, structured relevant and unstructured irrelevant sequences, and unstructured relevant and structured relevant sequences), phase 3 (switching with unstructured relevant sequences and irrelevant unstructured 


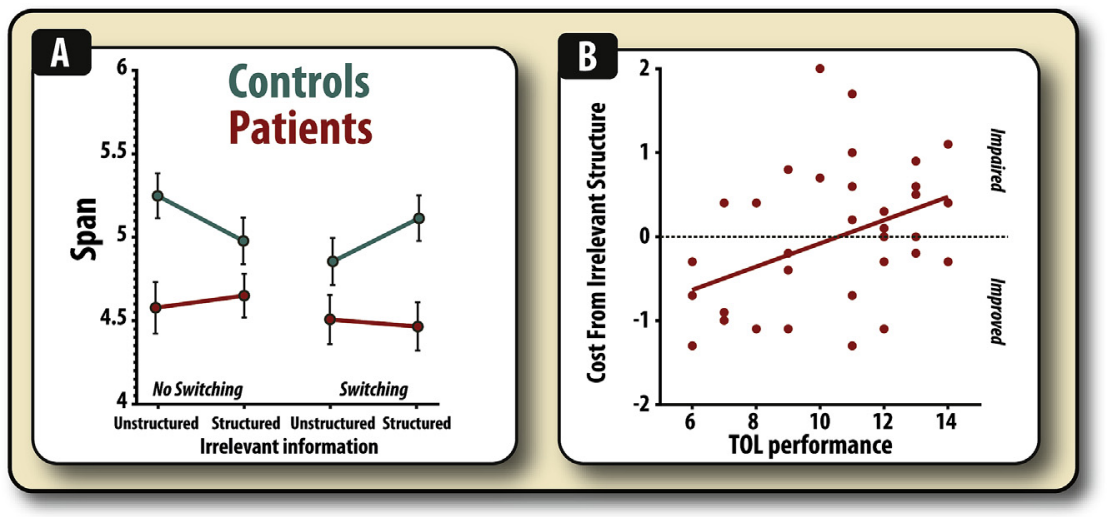

Fig. 3 - Span and switching. A) Spatial span for PD patients and controls in switching and non-switching contexts, split according to whether the irrelevant information was structured or unstructured. PD patients were unaffected by the experimental manipulation of structure in the irrelevant sequence or the need to switch. In contrast, controls were either aided (switching) or impaired (non-switching) by the presence of structured irrelevant information. Error bars reflect the standard error of the mean. B) Significant positive relationship between executive functioning (performance on the Tower of London; TOL) and the cost to span spatial incurred by irrelevant structure for PD patients only. The cost from irrelevant structure was calculated by subtracting spatial spans for the structured conditions (UN-STR and SW-UNSTR) from the unstructured irrelevant information conditions (UN-UN and SW-UNUN). A higher score on this metric indicates that spans decreased when ignoring structured irrelevant information.

sequences, and switching with unstructured relevant sequences and structured irrelevant sequences). There was a main effect of phase $[F(2,140)=14.91, p<.001]$, with patients and controls having progressively lower spans across the three phases, but, crucially, there was no significant interaction between time and disease $(F<1)$. Thus, there is no evidence that patients were declining (or fatiguing) more rapidly compared to controls.

\section{Discussion}

This study has revealed a new pattern of when, and under what conditions, PD patients show a reduction in their spatial span - the amount of information that can be held for a short period of time. A fundamental asymmetry in the way that PD patients deal with structure in relevant and irrelevant information was discovered. Structure in the relevant information normalises PD patients' spatial span, irrespective of executive performance level. The specific, dopamine-related, nature of this effect was underlined by the finding that the extent to which patients' performance improved with structured presentations was related to their level of medication. In contrast, these effects were not recapitulated in the case of dealing with structured irrelevant information - it was the patients' level of executive functioning that predicted whether they would show impaired or enhanced spatial spans.

\subsection{Chunking relevant information normalises WM in PD patients}

In the absence of encoding structured information, patients were generally found to show impaired spans compared to controls (Figs. 2A and 3A). Chunking is thought to be both an automatic and conscious process (Rossi-Arnaud, Pieroni, \&
Baddeley, 2006). That is, while chunking can occur automatically, information from long-term memory can also be recruited to impose structure on processed stimuli. Psychologically, the ability to chunk is thought to be facilitated by the episodic buffer, which together with the central executive, phonological loop and visuo-spatial sketch pad are thought to constitute the main components of WM (Baddeley, 2000). The episodic buffer is thought to facilitate the binding of information into chunks, and is itself controlled by executive processes, thus providing a conduit between working and long-term memory. This suggests that, in certain conditions, the psychological mechanisms that enable WM to take place retain their functionality in PD patients. Specifically, the fact that easy-to-chunk information normalised WM in patients implies that when the need to endogenously structure mental representations is bypassed, patients can store equivalent amounts of information as controls. However, deficits in the amount of information patients can store become readily apparent in the absence of that structure, suggesting that it is the inability to self-organise mnemonic material that lies at the core of patients' poor short-term recall.

The ability of structure in memoranda to act as a cognitive prosthesis in patients could stem from two, possibly related factors; a deficit in endogenously organising memoranda [applying top-down control (Cools, Miyakawa, et al., 2010)]; or that there may be increased neuronal noise in the parkinsonian brain that makes such control harder to execute (Bays, 2014; Manohar et al., 2015). Previous work has pointed to a dissociation in the neurocognitive origins of patients impairment on maintaining versus manipulating items in WM. Withdrawing patients from their dopaminergic state has no effect on the ease with which items can be maintained, but it does affect the manipulation of items in WM (Lewis et al., 2005). Within the present data there are also suggestions 
that the enhanced performance on conditions that contain structured information is present is related to patients' dopaminergic state. Patients who were taking the highest level of dopaminergic medication received the most benefit to their spans when recalling structured information. Although, equivalent levodopa dose is a poor measure of dopaminergic state - given that it is usually correlated with disease progression - there was no relationship between the UPDRS score (a marker of disease severity) and the performance gains from structure. Therefore, higher levels of dopamine appear to support a greater normalisation of spatial span by structure.

The results of this experiment differ slightly from a previous, similar study that used a smaller patient cohort (Gruszka et al., 2016). In addition to the possible differences in patient sample, there are also methodological differences between the two studies. In the previous study, participants were only tested on a fixed set size (four spatial locations). In the present study, however, participants were tested using a staircase (or ratchet) design, whereby the length of each spatial sequence would progressively increase. Thus, it is possible that the ratchet method is more sensitive at revealing differences between patients and controls. Indeed, larger differences between patients and controls have been found using this method (Fournet et al., 2000).

\subsection{Disease removes the sensitivity to switching and structured irrelevant information}

Contrary to previous studies that have found patients' impairments to be exacerbated in switching contexts (Cools et al., 2001; Fales et al., 2006; Hayes et al., 1998; Pollux, 2004; Ravizza \& Ciranni, 2002), their spatial spans were not modulated by the need to rapidly update their demarcation between relevant and irrelevant information (switching) or the introduction of salient, structured irrelevant information (Fig. 3B). Thus, this suggests that there may be limited impact of previously relevant - but now irrelevant - information on patients' short-term recall, and that previous studies that have shown patients to be disproportionately affected by these manipulations (Fallon, Hampshire, et al., 2016; Owen et al., 1993; Slabosz et al., 2006) may only have applicability in attentional set-shifting contexts.

Unlike patients, healthy controls' spans were significantly affected by the need to switch attention and whether structured irrelevant information was present. For controls, it appeared that the effect of structured irrelevant information on spans had opposite effects in switching and non-switching contexts. In a non-switching context, and thus when attention was singularly focused on one feature dimension, structured irrelevant information impaired spans. Yet, there was a trend for the reverse situation in switching contexts. Thus, the extent to which attention has to be switched, or updated, appears to modulate the effect that structured, potentially more salient, irrelevant information has on recall; in low updating context, salient irrelevant information is detrimental to performance, but in high updating contexts, salient irrelevant information can be beneficial, presumably because it becomes easier to ignore. Therefore, this finding demonstrates the heterogeneous nature of the effects irrelevant information can have on WM and that the level of updating, or switching, that occurs determines how structured (potentially salient) information is dealt with.

\subsection{Executive performance modulates the effect of irrelevant information in patients}

Although disease modulated the beneficial effect of structure on span, the same could not be said for structure in the irrelevant sequence. Rather, it was the level of executive performance, specifically in patients, that determined whether spans were improved or impaired by irrelevant structure. Patients with high executive functioning were impaired by irrelevant structure, whereas those with low executive performance were enhanced by irrelevant structure (Fig. 3B). Thus, patients with different levels of executive functioning seem to have opposite responses to structured irrelevant information. For high functioning patients, structured irrelevant information seems to attract attention in a way that is relatively detrimental to eventual recall. However, in low functioning patients, structured irrelevant information enhanced performance of patients, presumably by making irrelevant information easier to ignore.

In patients with high levels of executive performance, poor filtering of irrelevant information may have led to that information being cached in episodic buffer-like operations, ultimately producing a maladaptive form of chunking. That is, the failure to effectively filter irrelevant information may have led to the build-up of highly salient (distracting) information. Given that PD patients have been found to be impaired in ignoring salient information (Cools, Rogers, Barker, \& Robbins, 2010), this irrelevant information could have a greater capacity to interfere with mnemonic representations in PD patients, but seemingly only in those with high levels of executive performance.

Based upon the effects observed in the controls, we can make several hypotheses about the cognitive mechanisms underlying this process. For the controls, it was found that in switching contexts, irrelevant structure improved WM, whereas in non-switching contexts, irrelevant structure increased spans. Thus, the difference in performance according to executive functioning may have arisen due to a difference in the extent to which they mentally switch between the relevant and irrelevant sequences, i.e., high executive functioning patients are impaired by structure because they do not switch, whereas low executive functioning patients are improved because they do switch. Although plausible, further research, possibly with eye tracking or neural markers of attention, will be necessary to establish whether patients switch between the relevant and irrelevant sequences.

Despite this study's success in characterising which experimental manipulations influence spatial span performance in PD patients, this study has several limitations that constrain and qualify the inferences that can be made. Firstly, a key omission in this study is the lack of a condition where both the relevant and irrelevant sequences were spatially structured. As such, it remains to be determined whether, if both the relevant and irrelevant sequences were spatially structured, there would be a protective influence of spatial structure in the relevant information. 
Another limitation of this study is the extent to which it can comment upon how other forms of cognitive heterogeneity in patients modulate WM. Cognitive heterogeneity in PD is a multifaceted problem which has several dimensions (Kehagia et al., 2012). This study only assessed the role of one component of cognitive heterogeneity in patients - executive functioning as assessed by the TOL. As mentioned earlier, performance on this task is thought to be an indicator of fronto-striatal integrity (Lewis, Dove, et al., 2003; Nombela et al., 2014). It is likely that other indices of differential cognitive performance in patients - such as those that are dependent upon medial temporal lobe (MTL) or posterior cortical regions - could be seen to influence WM performance in patients. Indeed, patients with moderate Alzheimer's disease, a disorder associated with disruption to MTL regions, have been found to have a reduced ability to derive the mnemonic benefit from encoding structured sequences (Huntley et al., 2011). Future studies should evaluate whether other metrics of cognitive heterogeneity in PD patients affect patients' performance.

In summary, this study has, in a novel paradigm, confirmed previous suggestions that the capacity of medicated PD patients to encode new items into WM is limited, but crucially that this impairment can be normalised by having patients encoding structured memoranda. In contrast, the beneficial or detrimental effect of irrelevant information is highly dependent upon patients' level of executive functioning. By identifying the exact circumstances in which PD patients show impaired WM this study has further specified the precise cognitive substrates that require therapeutic intervention in PD patients.

\section{Supplementary data}

Supplementary data related to this article can be found at http://dx.doi.org/10.1016/j.cortex.2017.08.023.

\section{R E F E R E N C E S}

Aarsland, D., Bronnick, K., Williams-Gray, C., Weintraub, D., Marder, K., Kulisevsky, J., et al. (2010). Mild cognitive impairment in Parkinson disease A multicenter pooled analysis. Neurology, 75, 1062-1069.

Baddeley, A. (2000). The episodic buffer: A new component of working memory? Trends in Cognitive Sciences, 4, 417-423.

Baier, B., Karnath, H.-O., Dieterich, M., Birklein, F., Heinze, C., \& Müller, N. G. (2010). Keeping memory clear and stable-the contribution of human basal ganglia and prefrontal cortex to working memory. The Journal of Neuroscience, 30, 9788-9792.

Bays, P. M. (2014). Noise in neural populations accounts for errors in working memory. The Journal of Neuroscience, 34, 3632-3645.

Bor, D., Cumming, N., Scott, C. E., \& Owen, A. M. (2004). Prefrontal cortical involvement in verbal encoding strategies. European Journal of Neuroscience, 19, 3365-3370.

Bor, D., Duncan, J., Lee, A. C., Parr, A., \& Owen, A. M. (2006). Frontal lobe involvement in spatial span: Converging studies of normal and impaired function. Neuropsychologia, 44, 229-237.

Bor, D., Duncan, J., Wiseman, R. J., \& Owen, A. M. (2003). Encoding strategies dissociate prefrontal activity from working memory demand. Neuron, 37, 361-367.
Bor, D., \& Owen, A. M. (2007). A common prefrontal-parietal network for mnemonic and mathematical recoding strategies within working memory. Cerebral Cortex, 17, 778-786.

Brown, R. G., \& Marsden, C. D. (1988). Internal versus external cues and the control of attention in Parkinson's disease. Brain, 111, 323-345.

Bublak, P., Müller, U., Grön, G., Reuter, M., \& von Cramon, D. Y. (2002). Manipulation of working memory information is impaired in Parkinson's disease and related to working memory capacity. Neuropsychology, 16, 577.

Cools, R. (2006). Dopaminergic modulation of cognitive functionimplications for l-DOPA treatment in Parkinson's disease. Neuroscience and Biobehavioral Reviews, 30, 1-23.

Cools, R., Barker, R. A., Sahakian, B. J., \& Robbins, T. W. (2001). Mechanisms of cognitive set flexibility in Parkinson's disease. Brain, 124, 2503-2512.

Cools, R., Miyakawa, A., Sheridan, M., \& D'Esposito, M. (2010). Enhanced frontal function in Parkinson's disease. Brain, 133, 225-233.

Cools, R., Rogers, R., Barker, R. A., \& Robbins, T. W. (2010). Top-down attentional control in Parkinson's disease: Salient considerations. Journal of Cognitive Neuroscience, 22, 848-859.

Fales, C. L., Vanek, Z. F., \& Knowlton, B. J. (2006). Backward inhibition in Parkinson's disease. Neuropsychologia, 44, 1041-1049.

Fallon, S. J., Hampshire, A., Barker, R. A., \& Owen, A. M. (2016). Learning to be inflexible: Enhanced attentional biases in Parkinson's disease. Cortex. Retrieved from http://www. sciencedirect.com/science/article/pii/S0010945216301228.

Fallon, S. J., Zokaei, N., \& Husain, M. (2016). Causes and consequences of limitations in visual working memory. Annals of the New York Academy of Sciences. Retrieved from http://onlinelibrary.wiley.com/doi/10.1111/nyas.12992/full.

Fournet, N., Moreaud, O., Roulin, J. L., Naegele, B., \& Pellat, J. (2000). Working memory functioning in medicated Parkinson's disease patients and the effect of withdrawal of dopaminergic medication. Neuropsychology, 14, 247.

Gathercole, S. E., Brown, L., \& Pickering, S. J. (2003). Working memory assessments at school entry as longitudinal predictors of National Curriculum attainment levels. Educational and Child Psychology, 20, 109-122.

Gruber, A. J., Dayan, P., Gutkin, B. S., \& Solla, S. A. (2006). Dopamine modulation in the basal ganglia locks the gate to working memory. Journal of Computational Neuroscience, 20, 153-166.

Gruszka, A., Bor, D., Barker, R. R., Necka, E., \& Owen, A. M. (2016). The role of executive processes in working memory deficits in Parkinson's Disease. Polish Psychological Bulletin, 47, 123-130.

Hayes, A. E., Davidson, M. C., Keele, S. W., \& Rafal, R. D. (1998). Toward a functional analysis of the basal ganglia. Journal of Cognitive Neuroscience, 10, 178-198.

Huntley, J., Bor, D., Hampshire, A., Owen, A., \& Howard, R. (2011) Working memory task performance and chunking in early Alzheimer's disease. The British Journal of Psychiatry, 198, 398-403.

Kehagia, A. A., Barker, R. A., \& Robbins, T. W. (2012). Cognitive impairment in Parkinson's disease: The dual syndrome hypothesis. Neurodegenerative Diseases, 11, 79-92.

Kemps, E., Szmalec, A., Vandierendonck, A., \& Crevits, L. (2005). Visuo-spatial processing in Parkinson's disease: Evidence for diminished visuo-spatial sketch pad and central executive resources. Parkinsonism \& Related Disorders, 11, 181-186.

Lee, E.-Y., Cowan, N., Vogel, E. K., Rolan, T., Valle-Inclan, F., \& Hackley, S. A. (2010). Visual working memory deficits in patients with Parkinson's disease are due to both reduced storage capacity and impaired ability to filter out irrelevant information. Brain, awq197. 
Lewis, S. J., Cools, R., Robbins, T. W., Dove, A., Barker, R. A., \& Owen, A. M. (2003). Using executive heterogeneity to explore the nature of working memory deficits in Parkinson's disease. Neuropsychologia, 41, 645-654.

Lewis, S. J., Dove, A., Robbins, T. W., Barker, R. A., \& Owen, A. M. (2003). Cognitive impairments in early Parkinson's disease are accompanied by reductions in activity in frontostriatal neural circuitry. The Journal of Neuroscience, 23, 6351-6356.

Lewis, S. J., Slabosz, A., Robbins, T. W., Barker, R. A., \& Owen, A. M. (2005). Dopaminergic basis for deficits in working memory but not attentional set-shifting in Parkinson's disease. Neuropsychologia, 43, 823-832.

Manohar, S. G., Chong, T. T.-J., Apps, M. A., Batla, A., Stamelou, M., Jarman, P. R., et al. (2015). Reward pays the cost of noise reduction in motor and cognitive control. Current Biology, 25, 1707-1716.

McNab, F., \& Klingberg, T. (2008). Prefrontal cortex and basal ganglia control access to working memory. Nature Neuroscience, 11, 103-107.

Miller, G. A. (1956). The magical number seven, plus or minus two: Some limits on our capacity for processing information. Psychological Review, 63, 81.

Milner, B. (1971). Interhemispheric differences in the localization of psychological processes in man. British Medical Bulletin. Retrieved from http://psycnet.apa.org/psycinfo/ 1972-32010-001.

Monchi, O., Hanganu, A., \& Bellec, P. (2016). Markers of cognitive decline in PD: The case for heterogeneity. Parkinsonism \& Related Disorders, 24, 8-14.

Moustafa, A. A., Sherman, S. J., \& Frank, M. J. (2008). A dopaminergic basis for working memory, learning and attentional shifting in Parkinsonism. Neuropsychologia, 46, 3144-3156.

Nombela, C., Rowe, J. B., Winder-Rhodes, S. E., Hampshire, A., Owen, A. M., Breen, D. P., et al. (2014). Genetic impact on cognition and brain function in newly diagnosed Parkinson's disease: ICICLE-PD study. Brain, 137, 2743-2758.

Owen, A. M. (2004). Cognitive dysfunction in Parkinson's disease: The role of frontostriatal circuitry. The Neuroscientist, 10, 525-537.

Owen, A. M., Iddon, J. L., Hodges, J. R., Summers, B. A., \& Robbins, T. W. (1997). Spatial and non-spatial working memory at different stages of Parkinson's disease. Neuropsychologia, 35, 519-532.

Owen, A. M., Roberts, A. C., Hodges, J. R., Summers, B. A., Polkey, C. E., Robbins, T. W., et al. (1993). Contrasting mechanisms of impaired attentional set-shifting in patients with frontal lobe damage or Parkinson's disease. Brain, 116, 1159-1175.

Poewe, W., Berger, W., Benke, T. H., \& Schelosky, L. (1991). Highspeed memory scanning in Parkmson's disease: Adverse effects of levodopa. Annals of Neurology, 29, 670-673.

Pollux, P. M. (2004). Advance preparation of set-switches in Parkinson's disease. Neuropsychologia, 42, 912-919.

Postle, B. R., Jonides, J., Smith, E. E., Corkin, S., \& Growdon, J. H. (1997). Spatial, but not object, delayed response is impaired in early Parkinson's disease. Neuropsychology, 11, 171.

Ravizza, S. M., \& Ciranni, M. A. (2002). Contributions of the prefrontal cortex and basal ganglia to set shifting. Journal of Cognitive Neuroscience, 14, 472-483.

Rossi-Arnaud, C., Pieroni, L., \& Baddeley, A. (2006). Symmetry and binding in visuo-spatial working memory. Neuroscience, 139, 393-400.

Sawamoto, N., Piccini, P., Hotton, G., Pavese, N., Thielemans, K., \& Brooks, D. J. (2008). Cognitive deficits and striato-frontal dopamine release in Parkinson's disease. Brain, 131, 1294-1302.

Slabosz, A., Lewis, S. J., Smigasiewicz, K., Szymura, B., Barker, R. A., \& Owen, A. M. (2006). The role of learned irrelevance in attentional set-shifting impairments in Parkinson's disease. Neuropsychology, 20, 578.

Stoffers, D., Berendse, H. W., Deijen, J. B., \& Wolters, E. C. (2003). Deficits on Corsi's block-tapping task in early stage Parkinson's disease. Parkinsonism \& Related Disorders, 10, 107-111.

Tremblay, C., Achim, A. M., Macoir, J., \& Monetta, L. (2013). The heterogeneity of cognitive symptoms in Parkinson's disease: A meta-analysis. Journal of Neurology, Neurosurgery \& Psychiatry, 84, 1265-1272.

Williams-Gray, C. H., Mason, S. L., Evans, J. R., Foltynie, T., Brayne, C., Robbins, T. W., et al. (2013). The CamPaIGN study of Parkinson's disease: 10-year outlook in an incident population-based cohort. Journal of Neurology, Neurosurgery \& Psychiatry, 84, 1258-1264. 